03

\title{
Возбуждение вихревых течений на свободной поверхности жидкости вибрирующей пластиной
}

\author{
() В.А. Александров, С.П. Копысов, Л.Е. Тонков \\ Институт механики УдмФИЦ УрО РАН, \\ 426067 Ижевск, Россия \\ e-mail: ava@udman.ru
}

Поступило в Редакцию 7 июня 2018 г.

В окончательной редакции 30 января 201 г.

Принято к публикации 4 фревраля 2019 г.

Исследованы течения на поверхности жидкости при вибрациях в ней частично погруженной пластины. При низкочастотных вибрациях пластины на поверхности жидкости образуются по 2 вихря с каждой стороны поверхности пластины, в которых частицы жидкости движутся в направлении от поверхностей пластины. При параметрическом возбуждении поперечных капиллярных волн вблизи границы слоя жидкости, смачивающей поверхность пластины, направление течений в вихрях становится противоположным. Высокочастотные вибрации пластины создают на поверхности жидкости дополнительно вторичные вихри.

DOI: 10.21883/JTF.2019.07.47787.234-18

\section{Введение}

Исследованиям влияния вибраций на поведение жидкости со свободной поверхностью посвящено много работ. Проявление неустойчивости поверхности жидкости при вибрациях впервые было обнаружено Фарадеем в виде ряби, создаваемой параметрически возбуждаемыми двумерными капиллярными волнами [1]. Рябь Фарадея наблюдается в виде неподвижной решетки волн на свободной поверхности жидкости в вибрирующей емкости и на поверхности слоя жидкости на вибрирующей подложке. При возбуждении колебаний свободной поверхности жидкости в виде гравитационных, гравитационно-капиллярных и капиллярных волн частотой, в 2 раза меньшей частоты вибраций, в результате стробоскопического эффекта двумерные колебания поверхности жидкости становятся зрительно неподвижными.

Не менее интересными являются процессы возникновения течений в объеме жидкости при вибрациях. Периодически распределенные внутренние течения в жидкости исследованы в [2] при возбуждении гравитационных волн Фарадея в водном растворе бромида калия толщиной $20 \mathrm{~mm}$ в прямоугольной емкости из прозрачного оргстекла размером $190.5 \times 25.4 \times 69.6 \mathrm{~mm}$. Вертикальные вибрации емкости с частотой в интервале $7-11 \mathrm{~Hz}$ возбуждают на поверхности жидкости волны Фарадея с частотой в 2 раза менышей частоты вибраций, и внутри вибрирующей жидкости формируются периодически распределенные вихревые течения.

Колебания свободной поверхности жидкости с увеличением частоты вибраций обусловливаются силами тяжести и поверхностным натяжением и эти колебания возбуждаются в виде гравитационно-капиллярных волн. В работе [3] наблюдали распределенные течения на поверхности дистиллированной воды в емкости цилиндрической формы диаметром $65 \mathrm{~mm}$ и глубиной $10 \mathrm{~mm}$ и квадратной формы стороной $50 \mathrm{~mm}$ и глубиной $10 \mathrm{~mm}$ при вертикальных вибрациях емкости в интервале частот $20-50 \mathrm{~Hz}$. Возникновение течений авторы связывают с возбуждением пар волн с неколлинеарными волновыми векторами, распространяющихся по поверхности жидкости.

Вихревые течения возникают также и в вибрирующих пленках жидкости. Устойчивость к поперечным колебаниям проявляют мыльные пленки воды, которые и применяются в экспериментах. Возбуждение течений в мыльных пленках круглой и квадратной форм низкочастотными вибрациями в интервале от 20 до $200 \mathrm{~Hz}$ показано в [4]. Для этого пленка устанавливалась горизонтально в полости емкости, которая помещалась на вибростенд. Возникновение течений в мыльной пленке авторы объясняют возбуждением волн Марангони, которые представляют собой упругие продольные волны в пленке. Эти волны вследствие вязкости захватывают подлежащий пограничный слой жидкости, приводя его в движение. Эффект Марангони связан с изменением поверхностного натяжения, и движение в жидкости вызвано касательными градиентами поверхностного натяжения [5]. Вибрации большой амплитуды могут привести к периодическому изменению поверхностного натяжения жидкости и нелинейному возбуждению волн на ее свободной поверхности. В работе [6] на механизм течений указывают как на усредненное действие капиллярных колебаний на жидкость, рассматривая эти течения как акустические, вызванные радиационными силами.

В работе [7] были описаны поверхностные вихревые течения в слоях и каплях жидкости на вибрирующей гибкой пластине, возбужденной пьезоэлектрическим преобразователем. Установлено, что общий поток примыкающих вихревых течений в каплях и в слое жидкости на вибрирующей пластине оказывается направленным поперек гребней стоячей капиллярной волны, возбужда- 
емой на свободной поверхности жидкости. В стоячей поверхностной волне растягивающие силы сосредоточены в узлах колебаний, направлены перпендикулярно гребням волн и имеют проекции на плоскости неподвижной свободной поверхности.

Несмотря на многочисленные результаты исследований, механизм образования течений в жидкости при вибрациях до конца не выяснен. Так как при возникновении течений на поверхности жидкости одновременно наблюдаются в зависимости от частоты вибраций гравитационно-капиллярные или капиллярные колебания в виде волн, по-видимому, необходимо учитывать вклад продольной составляющей этих волн.

Целью настоящей работы является экспериментальное исследование возбуждения капиллярных колебаний вибрирующей пластиной и условий возникновения течений на поверхности жидкости.

\section{Постановка экспериментов}

Схема экспериментального устройства приведена на рис. 1. Устройство содержит емкость с жидкостью, генератор звуковой частоты, усилитель, электромеханический преобразователь и присоединенную к нему через волновод пластину. Преобразователь с пластиной устанавливается на штативе через держатель так, что пластина находится в контакте со свободной поверхностью жидкости в емкости. Генератор с усилителем возбуждает в преобразователе вибрации, которые через волновод передаются пластине и жидкости. Для получения низкочастотных вибраций пластины в качестве электромеханического преобразователя использовались электродинамические громкоговорители, к центру диафрагмы которых пластина приклеивалась через волновод из отрезка медного толстого провода. Для возбуждения высокочастотных колебаний упругого элемента использовались пьезоэлектрические преобразователи - пьезоизлучатели FML-20T-6.0A1-100, FML-20T-4.5A1-100, FML-40T-1.0A1-100 и 3П-4. Короткая пластина приклеивалась к корпусу пьезоизлучателя через волновод из

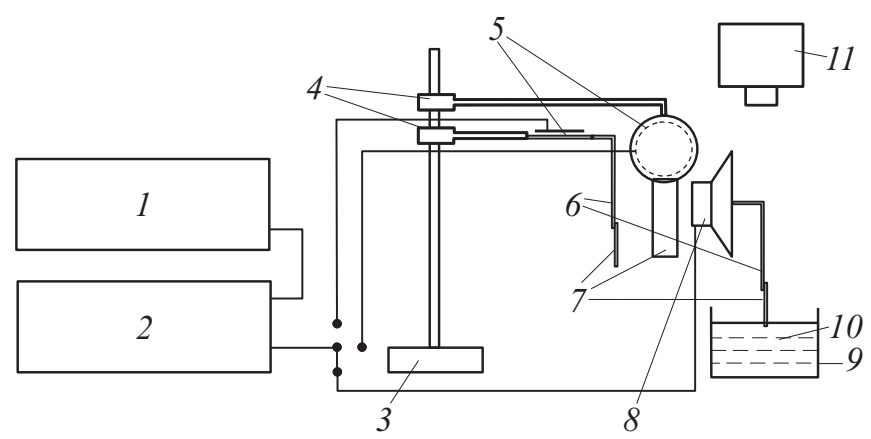

Рис. 1. Схема устройства: 1 - генератор сигналов, $2-$ усилитель, 3 - штатив, 4 - держатель, 5 - пьезоизлучатель, 6 - волновод, 7 - пластина, 8 - громкоговоритель, $9-$ емкость, 10 - жидкость, 11 - цифровая камера. стальной или медной проволоки. Длинная пластина приклеивалась к корпусу пьезоизлучателя непосредственно коротким краем. В части экспериментов колебания на поверхности жидкости возбуждались вибрирующим стержнем.

Гидродинамические процессы, создаваемые в жидкости вибрациями пластины, наблюдались с помощью микроскопа JW-15T, оснащенного цифровой камерой фирмы OMAX A35140U3, цифровым фотоаппаратом Canon EOS 650D и записывались на компьютер. В качестве жидкости использовалась дистиллированная вода. Визуализация течений осуществлялась мелкодисперсным порошком графита, люминофора и серы $(0.01-0.1) \mathrm{mm}$.

\section{Результаты и их обсуждение}

Исследования показали следующее. При подведении края пластины к свободной поверхности жидкости и частичном погружении пластины в жидкость происходит капиллярный подъем жидкости в результате смачивания поверхности пластины. С возбуждением вибраций пластины в виде поперечных колебаний капиллярный подъем жидкости увеличивается. Вибрирующая пластина возбуждает поверхностные колебания жидкости, распространяющиеся в направлении от поверхности пластины в виде капиллярных волн. Мелкодисперсные частицы, засеянные на поверхность жидкости, при возбуждении вибраций пластины приобретают движение по замкнутым траекториям, указывая на возникновение поверхностных вихревых течений. На рис. 2, а показана картина течений на поверхности воды в цилиндрической кювете диаметром $29 \mathrm{~mm}$ и высотой $9 \mathrm{~mm}$, создаваемая РЕТ-пластиной $12.0 \times 10.0 \times 0.25 \mathrm{~mm}$, вибрирующей в поперечном направлении частотой $230 \mathrm{~Hz}$. От каждой стороны поверхности пластины создаются два вихревых течения, в которых частицы движутся в противоположных направлениях. Вдоль линии в направлении колебаний, перпендикулярной плоскости пластины в еe середине, вихревые течения имеют одинаковое направление и частицы движутся в общем потоке. Вихревые течения создаются также и колебаниями стержня на свободной поверхности жидкости. Направления колебаний стержня могут быть как в плоскости свободной поверхности жидкости, так и нормальными к поверхности жидкости. В качестве примера на рис. 2, $b$ представлена фотография течений на поверхности воды, создаваемых участком стержня диаметром $0.6 \mathrm{~mm}$ и длиной $8 \mathrm{~mm}$, вибрирующим с частотой $737 \mathrm{~Hz}$. На изображениях рис. 2 течения визуализируются частицами люминофора. Примечательно, что в каждом устоявшемся плоском вихревом течении наблюдаются места сужения и расширения потока, в которых скорость перемещения частиц и их плотность отличаются. В особенности выделяются участки поверхности жидкости, симметрично расположенные по обеим сторонам поверхности пластины или стержня, где частицы движутся с ускорением. 


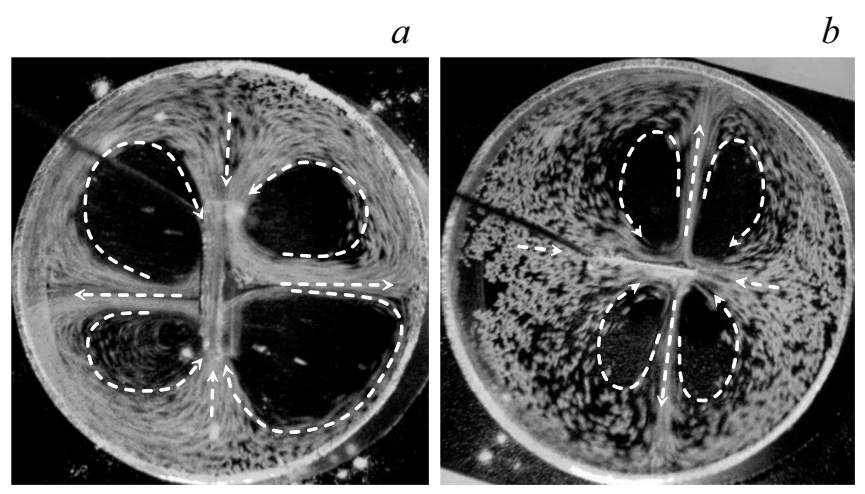

Рис. 2. Вихревые течения на поверхности воды, создаваемые пластиной $(a)$ и стержнем $(b)$ в цилиндрической емкости.

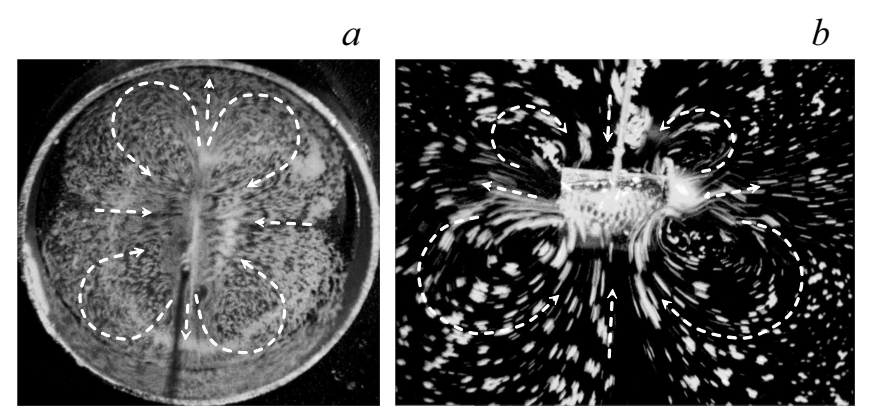

Рис. 3. Вихревые течения на поверхности воды, создаваемые вертикальной $(a)$ и наклонной $(b)$ пластиной в условиях возбуждения поперечной капиллярной стоячей волны вблизи границы смачивающего слоя.

При погружении пластины в жидкость на минимальную глубину, при которой на поверхности пластины образуется смачивающий слой жидкости, или погружении пластины в жидкость под углом с увеличением амплитуды колебаний пластины в некоторых интервалах частот вибраций наблюдается изменение направления движения течений - общий поток в парах вихрей становится направленным в сторону поверхности пластины (рис. 3). При этом наблюдается возбуждение капиллярных колебаний в виде поперечных стоячих волн на поверхности слоя жидкости, смачивающего пластину и дополнительный капиллярный подъем жидкости по поверхности пластины. Гребни стоячей волны являются поперечными к границе линии смачивания. На рис. 3 представлены плоские течения, визуализируемые соответственно частицами люминофора и серы, и периодическая структура капиллярных волн на поверхности слоя воды, смачивающей поверхность вертикально расположенной пластины при частоте вибраций $602 \mathrm{~Hz}$ и пластины, частично погруженной под углом $45^{\circ}$ и вибрирующей с частотой $1.02 \mathrm{kHz}$. Картина капиллярных колебаний в виде стоячей волны на поверхности жидкости, смачивающей вертикально установленную вибрирующую пластину вблизи межфазной границы, показана на рис. 4. Край пластины погружен на глубину
$0.5 \mathrm{~mm}$ до образования смачивающего слоя жидкости на поверхности пластины. На свободной поверхности воды вдали от пластины формируется интерференционная картина, создаваемая волнами, распространяющимися в направлении нормали и в направлении, параллельной плоскости поверхности пластины. Течения на поверхности в данном случае не заметны из-за прозрачности воды и отсутствия частиц на поверхности воды.

При вибрациях пластины в жидкости в интервале высоких звуковых частот и возбуждении поперечных капиллярных волн на краю смачивающего слоя жидкости скорость вихревых течений вблизи пластины увеличивается. Вследствие этого на свободной жидкости создаются вторичные течения. При этом количество вихрей на поверхности жидкости увеличивается, их размеры уменьшаются.

На изображения течений существенно влияют природа, размер и концентрация частиц, которые применяются для визуализации потоков. Тяжелые частицы со временем оседают на дно кюветы и искажают картину возбуждаемых течений. Слишком большая концентрация частиц не позволяет использовать компьютерную обработку получаемых видеоизображений. Фон и освещенность также влияют на качество изображений. Поэтому на белом фоне лучше использовать темные частицы, например графита, а на черном фоне - частицы белого или желтого цветов. Примером удачного изображения является рис. $3, b$, который получен на черном фоне с использованием порошка частиц серы ярко-желтого цвета. Покадровая обработка цифрового видеоизображения течений в данном эксперименте в программном пакете PIVlab позволила получить векторные поля скоростей в течениях (рис. 5).

По шкале скоростей можно оценить скорости движения частиц и соответственно распределение скоростей в вихревых течениях. В частности, для данного эксперимента значения скоростей находятся в пределах $2-20 \mathrm{~mm} / \mathrm{s}$. По полю скорости также были построены поля завихренности, дивергенции скорости и линии тока (рис. 6), что позволяет определить интенсивность и

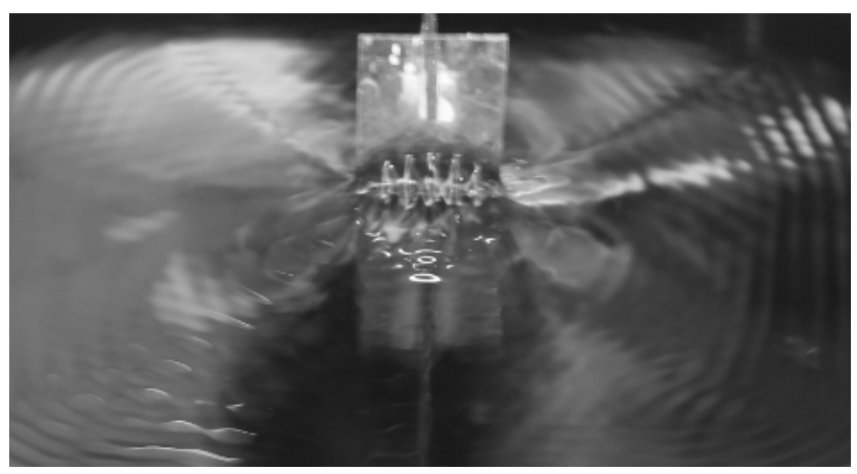

Рис. 4. Интерференционная картина волнового поля на свободной поверхности воды при параметрическом возбуждении двумерных капиллярных колебаний пластиной, вибрирующей с частотой $281 \mathrm{~Hz}$. 


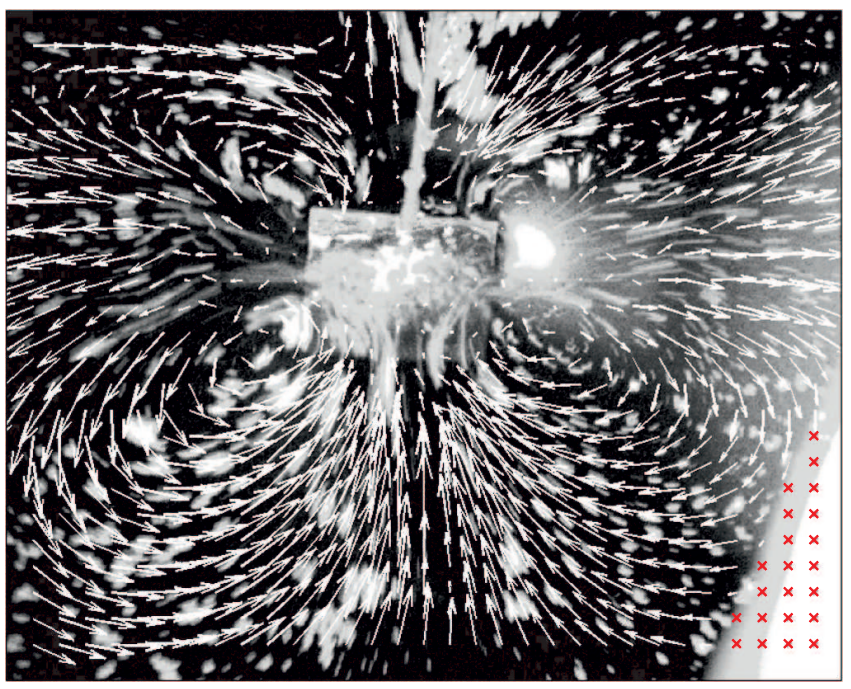

Рис. 5. Векторное поле скорости течения, создаваемого пластиной, вибрирующей с частотой $1.02 \mathrm{kHz}$.

положение центров вихрей, а также количественно оценить по величине дивергенции (рис. 6, $b$ ) погрешность восстановления поля скорости.

Процессы возбуждения капиллярных колебаний и волн вибрирующей пластиной на поверхности воды исследовались с помощью стальной пластины $14.0 \times 3.6 \times 0.1 \mathrm{~mm}$, вибрации которой возбуждались громкоговорителем при относительно низких звуковых частотах, при которых в пластине еще не возбуждаются вибрации с собственной частотой.

На рис. 7, $a-c$ показаны формы поверхности объема воды вблизи межфазной границы с пластиной, наблюдаемых в направлении к нормали пластины соответственно при отсутствии вибраций, при вибрациях с частотой $50 \mathrm{~Hz}$ и вибрациях с частотой $372 \mathrm{~Hz}$ с возбуждением поперечной капиллярной волны. На рис. $7, d-f$ показаны формы поверхности воды, наблюдаемых со стороны края пластины соответственно при отсутствии вибраций, при вибрациях с частотой $400 \mathrm{~Hz}$ и вибрациях с частотой $400 \mathrm{~Hz}$ при параметрическом возбуждении поперечной капиллярной волны. На изображениях наблюдается дополнительный подъем смачивающего слоя жидкости по поверхности вибрирующей пластины относительно положения границы смачивания на поверхности неподвижной пластины. На рис. 7, $c, f$ присутствуют гребни поперечной капиллярной стоячей волны.

Для выяснения роли капиллярных волн в образовании течений на свободной поверхности жидкости наблюдали за процессом формирования капиллярных колебаний в воде пластиной, вибрирующей с частотой $50 \mathrm{~Hz}$. При этом фиксировали изменение формы капиллярно приподнятого объема воды в плоскости пластины за период колебаний. На рис. 8, а изображена форма капиллярно приподнятого объема воды по поверхности неподвижной пластины, частично погруженной в воду в вертикальном положении. Изображения на рис. $8, b$ и $d$ соответствуют крайним правому и левому положениям пластины, в которых скорость пластины равна нулю. Форма поверхности капиллярно приподнятого объема воды на разных сторонах пластины является различной, а изображения в целом являются практически зеркально симметричными. Изображения на рис. 8,c и $e$ соответствуют положениям пластины, в которых пластина движется влево и вправо с максимальной скоростью. На фотографиях видно, что высота объема воды снижается, а свободный край смещается в противоположную сторону от направления от движения пластины. Жидкость приподнятого объема воды при этом растекается вдоль свободной поверхности. Заметно, что смещение свободного края растекающегося объема воды почти в 3 раза превышает смещение пластины за четверть периода колебаний. На изображения рис. 8,c и $e$ наложены короткая и длинная стрелки, указывающие смещение пластины и края растекающегося объема жидкости. Очевидно, что край растекающегося объема воды движется вдоль горизонтальной поверхности воды со скоростью капиллярной волны, превышающей скорость движения пластины.

Из дисперсионного соотношения, связывающего круговую частоту и волновое число гравитационно-капиллярных волн [3] , для глубокой жидкости можно получить следующее уравнение для скорости $c$ и частоты $f$ волн:

$$
2 \pi f c-g=(\sigma / \rho)\left(4 \pi^{2} f^{2} / c^{2}\right),
$$

где $\sigma$ - поверхностное натяжение и $\rho-$ плотность жидкости. Решая это уравнение для частоты $50 \mathrm{~Hz}$, находим, что скорость капиллярных волн в воде составляет $(0.28-0.29) \mathrm{m} / \mathrm{s}$. В данном эксперименте при амплитуде нормальных колебаний $0.5 \mathrm{~mm}$ амплитуда скорости пластины равна $0.16 \mathrm{~m} / \mathrm{s}$.

С целью выяснения механизма возбуждения капиллярных колебаний в жидкости частично погруженной в нее вибрирующей пластиной проведем анализ изменения давления в жидкости, создаваемой пластиной вблизи ее поверхности. Пусть пластина совершает колебания в направлении вдоль свободной поверхности жидкости. Если рассматривать движущийся элемент пластины в жидкости, то при его движении в сторону жидкости выталкивается некоторый объем жидкости, равный произведению площади элемента поверхности пластины и смещения этого элемента в колебательном движении в жидкости. В случае несжимаемой жидкости при движении в ней пластины жидкость переместится с фронтальной поверхности к противоположной тыльной поверхности, огибая края пластины. При вибрациях пластины противоположные поверхности пластины периодически изменяют направление движения по отношению к жидкости, в результате которого в каждый полупериод колебаний одна из них выталкивает жидкость, другая тянет жидкость за собой. Область взаимодействия элемента пластины с жидкостью при этом определяется толщиной пластинки 


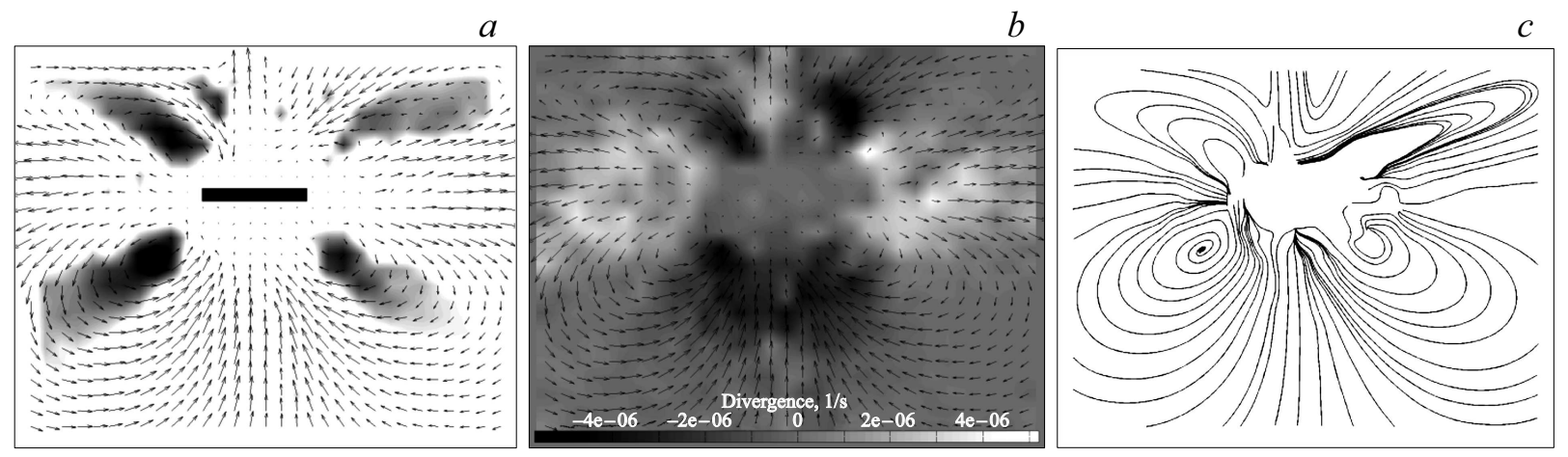

Рис. 6. Вихри $(a)$, дивергенция $(b)$ и линии тока $(c)$ в течениях.

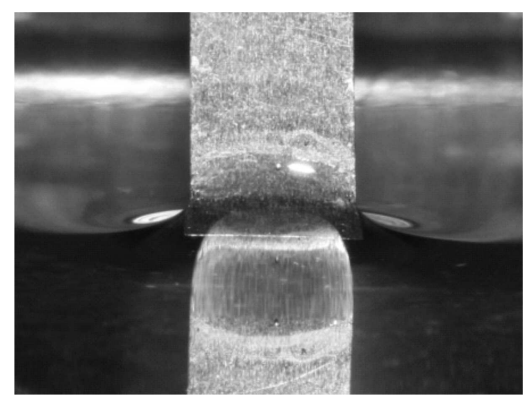

$d$

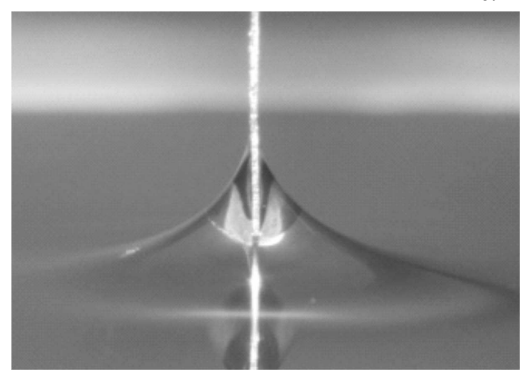

b

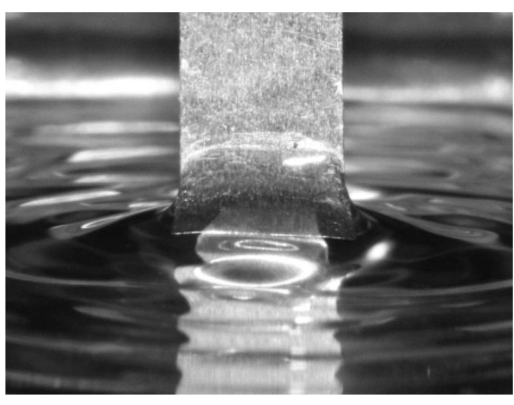

e

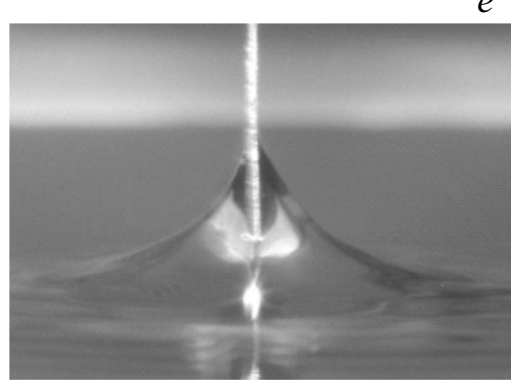

c

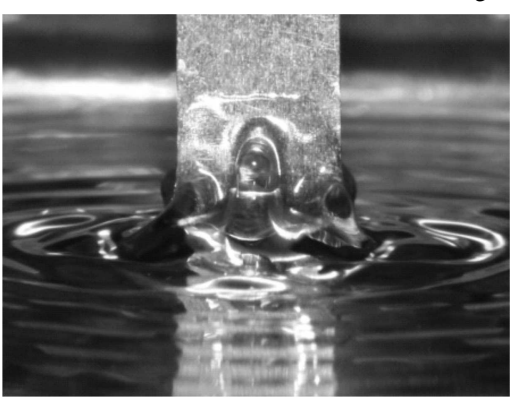

f

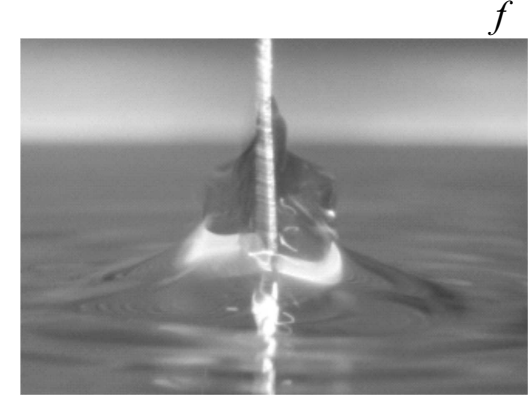

Рис. 7. Капиллярный подъем воды по поверхности вертикально погруженной стальной пластины: $a-$ без вибраций, $b-$ при вибрациях $50 \mathrm{~Hz}, c-$ при вибрациях $372 \mathrm{~Hz}, d-$ без вибраций, $e-$ при вибрациях $400 \mathrm{~Hz}, f-$ при вибрациях $400 \mathrm{~Hz}$ с параметрическим возбуждением поперечных капиллярных колебаний.

и амплитудой колебаний этого элемента при вибрациях пластины.

Пусть с началом отсчета времени элемент поверхности $\Delta S$ пластины движется в сторону жидкости в положительном направлении. Тогда колебания этого элемента поверхности пластины можно записать соотношением

$$
s=-s_{0} \cos \omega t
$$

где $s$ - смещение элемента поверхности пластины от положения равновесия, $s_{0}$ - амплитуда и $\omega$ - частота колебаний, равная частоте вибраций пластины. Скорость движения элемента поверхности пластины при этом равна

$$
d s / d t=\omega s_{0} \sin \omega t .
$$

Элементарный объем жидкости, вытесненный движущимся элементом поверхности пластины в момент времени $t$ от начала отсчета, составит

$$
V=\Delta S s_{0}(1-\cos \omega t) \text {. }
$$

Масса этого объема жидкости равна

$$
m=\rho \Delta S s_{0}(1-\cos \omega t),
$$

где $\rho-$ плотность жидкости. Так как в начальный момент времени скорость элемента поверхности пластины равна нулю, импульс, переданный пластиной этому объему жидкости, равен

$$
P=(\rho / 2) \Delta S \omega s_{0}^{2}(1-\cos \omega t) \sin \omega t .
$$

Давление этой жидкости на пластину можно определить из равенства $p=(1 / \Delta S) d P / d t$, определяющего давление сил инерции жидкости на элемент поверхности 


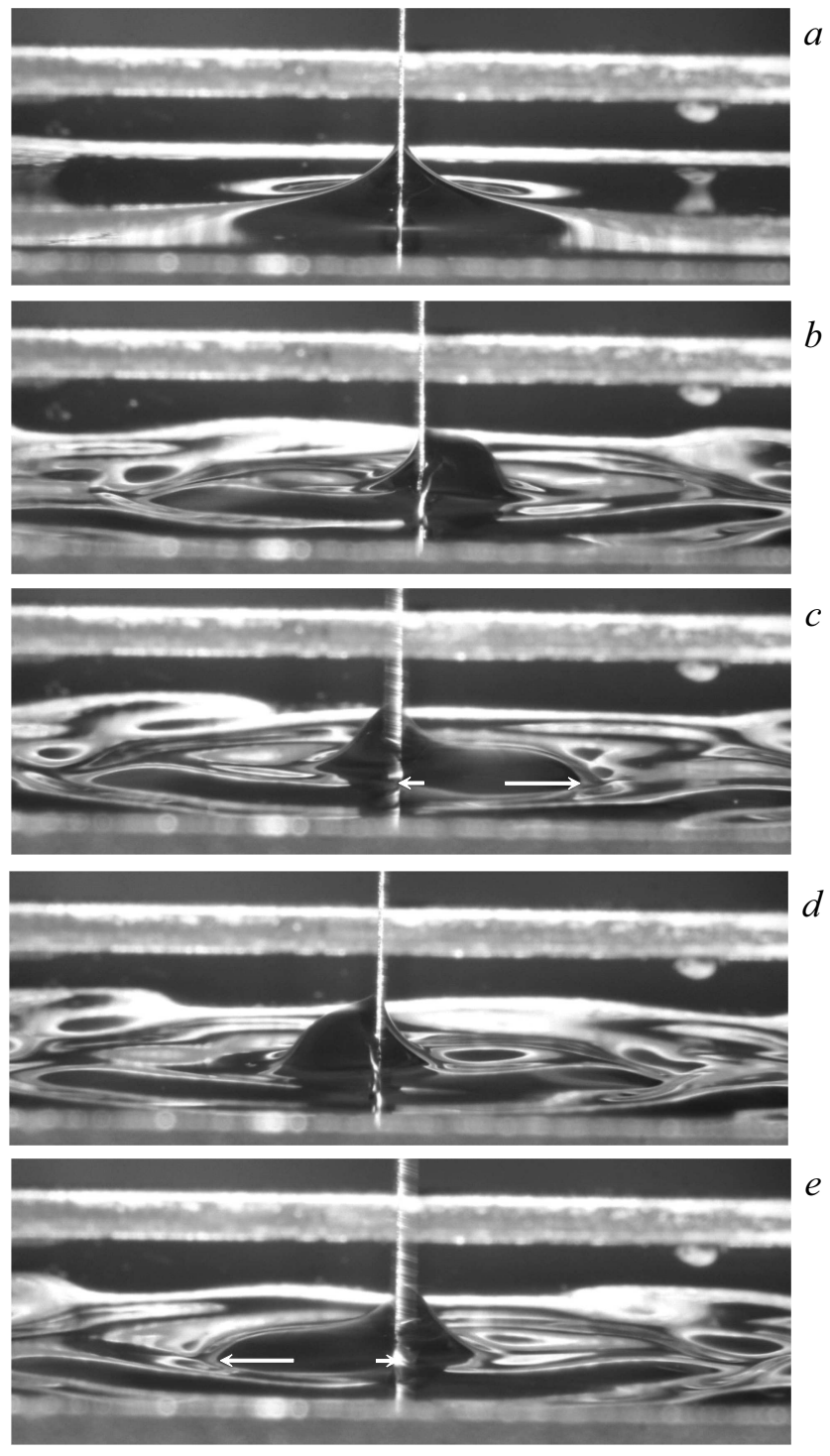

Рис. 8. Капиллярный подъем воды, смачивающей поверхность неподвижной вертикальной стальной пластины $(a)$, и форма объема жидкости вблизи пластины и волнового поля на свободной поверхности воды при различных фазах нормальных колебаний с частотой $50 \mathrm{~Hz}: b-\omega t=(2 n+1) \pi ; c-$ $\omega t=(4 n+3) \pi / 2 ; d-\omega t=(2 n+2) \pi ; e-\omega t=(4 n+5) \pi / 2$.

пластины. Тогда выражение для давления инерционных сил жидкости на пластину в направлении колебаний можно записать в виде

$$
p=(\rho / 2) \omega^{2} s_{0}^{2}(\cos \omega t-2 \cos \omega t) .
$$

Жидкость при движении в ней пластины вытесняется со скоростью движения элемента поверхности пластины и приобретает удельную энергию, пропорциональную квадрату скорости. В движущейся жидкости удельная энергия определяет гидродинамическое давление, на величину которого давление меньше по отношению к неподвижной жидкости. Тогда давление, создаваемое в жидкости участком поверхности вибрирующей пластины, равно

$$
p=(\rho / 2) \omega^{2} s_{0}^{2}\left(\cos \omega t-2 \cos \omega t-\sin ^{2} \omega t\right) .
$$

Это выражение можно переписать в виде

$$
p=(\rho / 2) \omega^{2} s_{0}^{2}\left(\cos \omega t-\cos ^{2} \omega t\right) .
$$

Давление в жидкости вблизи вибрирующей пластины отличается от давления в неподвижной жидкости вдали от пластины на величину гидродинамического давления, обеспечивающего переток жидкости с фронтальной на тыльную поверхность пластины. Так как скорость жидкости при этом равна скорости перемещения поверхности пластины, гидродинамическое давление жидкости на краях пластины равно

$$
p=(\rho / 2) \omega^{2} s_{0}^{2} \sin ^{2} \omega t .
$$

Преобразование этого выражения к равенству

$$
p=(\rho / 4) \omega^{2} s_{0}^{2}(1-\cos 2 \omega t)
$$

указывает, что гидродинамическое давление в жидкости вблизи краев пластины имеет постоянную и переменную составляющие. Так как величина переменной составляющей в среднем за период колебаний равна нулю, то вблизи краев вибрирующей пластины гидродинамическое давление в среднем равно постоянной составляющей $p=(\rho / 4) \omega^{2} s_{0}^{2}$. На рис. 9 представлены кривые зависимости давления, создаваемые вибрирующей пластиной, от фазы колебаний при нормированной амплитуде давления $p_{0}=(\rho / 2) \omega^{2} s_{0}^{2}=1$. Как видно из этих зависимостей, давление в жидкости вблизи поверхности вибрирующей пластины является импульсным с отрицательным с минимальным значением $p=-\rho \omega^{2} s_{0}^{2}$ при значениях фаз колебаний элемента поверхности пластины $\omega t=(2 n+1) \pi,(n=0,1,2 \ldots)$. Кривая зависимости давления, создаваемого в жидкости противоположной поверхностью пластины, сдвинута по фазе на $\pi$.

Рассмотрим поведение жидкости со свободной поверхностью при взаимодействии с частично погруженной

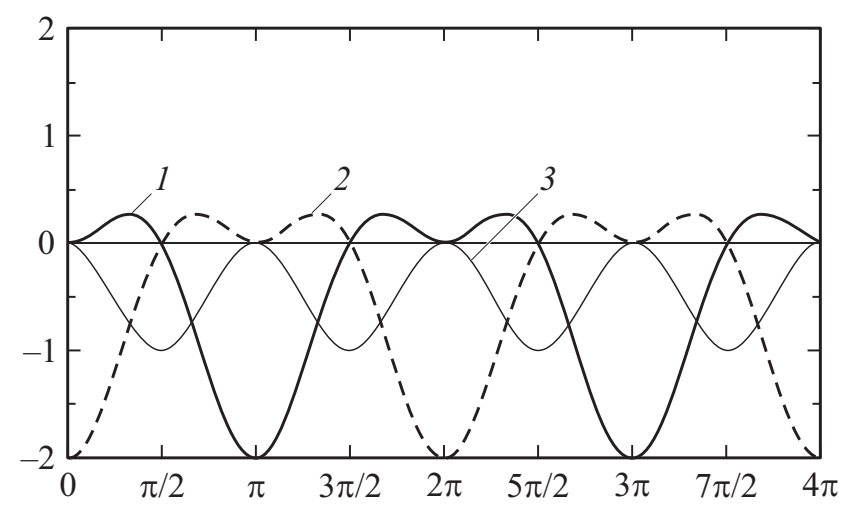

Рис. 9. Зависимости давления в жидкости вблизи вибрирующей пластины от фазы колебаний: 1,2 - на поверхностях пластины, 3 - на краях пластины. 
пластиной. Если выделить некоторый объем жидкости вблизи межфазной границы, то этот объем окажется ограниченным с двух смежных сторон поверхностями раздела жидкость-твердое тело и жидкость-газ (воздух атмосферы). Остальные стороны выделенного объема являются общими с окружающей жидкостью. В силу несжимаемости жидкости подвижность поверхности раздела жидкость-твердое тело вместе с нормальными колебаниями пластины придает подвижность свободной поверхности жидкости вблизи поверхности пластины.

Смачиваемость поверхности пластины приводит к образованию мениска жидкости с искривленной свободной поверхностью и капиллярному подъему жидкости вблизи межфазной границы. Капиллярный подъем вызван тем, что давление Лапласа под вогнутой поверхностью жидкости вблизи пластины является отрицательным и это давление компенсируется давлением приподнятой части жидкости над горизонтальной поверхностью жидкости. Полное давление на искривленной поверхности жидкости равно атмосферному давлению так же, как и на ее горизонтальной поверхности.

$\mathrm{C}$ возбуждением вибраций пластины давления в жидкости, связанное с ускоренным движением участка поверхности пластины, изменяется с частотой вибраций. В моменты времени, соответствующие началу торможения поверхности пластины, давление становится отрицательным и принимает максимальное отрицательное значение в моменты времени, соответствующие изменению направления движения пластины, когда ее скорость равна нулю. Жидкость при этом стремится в область отрицательного давления. Наличие свободной поверхности вблизи пластины приводит к увеличению площади поверхности жидкости за счет изменения ее кривизны и относительному подъему объема жидкости под искривленной поверхностью, что подтверждается изображениями на рис. $8, b$ и $d$. Соответственно при этом часть кинетической энергии жидкости преобразуется в потенциальную энергию искривленной поверхности вблизи пластины. В дальнейшем с началом движения пластины в обратном направлении потенциальная энергия искривленной поверхности жидкости переходит в кинетическую энергию движения растекающегося объема жидкости, а деформация свободной поверхности распространяется в виде цилиндрической капиллярной волны (рис. $8, c$ и $d$ ).

Дисперсионное соотношение для скорости капиллярной волны и волнового числа можно представить в виде

$$
c_{c}^{2}=(\sigma / \rho) k
$$

Так как приближенно считается, что длина $\lambda$ капиллярной волны пропорциональна радиусу $R$ кривизны поверхности и равна $\lambda=2 \pi R$, то $k=1 / R$. В таком приближении волновой вектор в капиллярной волне по модулю оказывается равным кривизне свободной поверхности в гребнях волны. Тогда $c_{c}^{2}=(\sigma / \rho R)=p_{L} / \rho$, где $p=\sigma / R$ - избыточное давление Лапласа в капиллярной волне. Отсюда скорость капиллярной волны

$$
c_{c}^{2}=(\sigma / \rho R)^{1 / 2}=\left(p_{L} / \rho\right)^{1 / 2} .
$$

Из дисперсионного соотношения можно получить также выражения для плотности энергии и вектора Умова - вектора плотности потока энергии в капиллярной волне соответственно в виде

$$
\begin{gathered}
\rho c_{c}^{2}=\sigma k=\sigma / R, \\
\mathbf{S}_{c}=\left(\rho c_{c}^{2}\right) \mathbf{c}_{c}=(\sigma / R) \mathbf{c}_{c} .
\end{gathered}
$$

Вектор Умова в капиллярной волне равен по величине плотности потока энергии

$$
S_{c}=\omega \sigma=(1 / \rho)^{1 / 2}(\sigma / R)^{3 / 2} .
$$

Плотность импульса капиллярной волны в жидкости составит

$$
p_{c}=\rho c_{c}=\left(\rho p_{L}\right)^{1 / 2} .
$$

С учетом радиуса кривизны поверхности

$$
p_{c}=(\rho \sigma / R)^{1 / 2} \text {. }
$$

Источником капиллярных волн является возмущение поверхности жидкости, создаваемое пластиной непосредственно вблизи поверхности. Из проведенных экспериментов, представленных на рис. $7, b$ и $b$, видно, что поверхность капиллярно приподнятого объема жидкости вблизи пластины является трехмерной и имеет два радиуса кривизны $R_{x}$ и $R_{y}$ в сечениях соответственно продольной и поперечной к плоскости колебаний пластины.

Для скорости вектора Умова и плотности импульса в двумерной капиллярной волне, распространяющейся от объема жидкости с разными радиусами кривизны свободной поверхности, можно записать следующие выражения:

$$
\begin{gathered}
c_{c}^{2}=c_{c x}^{2}+c_{c y}^{2}=(\sigma / \rho)\left(1 / R_{x}+1 / R_{y}\right), \\
S_{c}^{2}=S_{c x}^{2}+S_{c y}^{2}=\sigma^{2}\left(\omega_{x}^{2}+\omega_{y}^{2}\right)=\left(\sigma^{3} / \rho\right)\left(1 / R_{x}^{3}+1 / R_{y}^{3}\right), \\
p_{c}^{2}=p_{c x}^{2}+p_{c y}^{2}=\rho \sigma\left(1 / R_{x}+1 / R_{y}\right) .
\end{gathered}
$$

При этом волновой вектор капиллярной волны может быть представлен в виде

$$
k^{2}=k_{x}^{2}+k_{y}^{2}=1 / R_{x}^{2}+1 / R_{y}^{2} .
$$

Так как в эксперименте $R_{x}$ меньше, чем $R_{y}$, составляющие скорости, векторы Умова, плотности импульса и волнового вектора капиллярной волны, распространяющейся от капиллярно приподнятого пластиной объема жидкости, в направлении в плоскости колебаний пластины оказываются больше по сравнению с их составляющими в поперечном направлении колебаний пластины. 
Таким образом, капиллярные волны, исходящие от периодически возмущенной поверхности жидкости вблизи вибрирующей пластины в ближней зоне, распространяются с различными составляющими скорости и волнового вектора вследствие разной кривизны в ортогональных плоскостях. При передаче импульса поверхностному слою жидкости от капиллярной волны с различными составляющими в поперечных направлениях перемещение слоя жидкости происходит с разными скоростями в этих направлениях, что и наблюдается в эксперименте с ускоряющимся поверхностным потоком в направлении плоскости колебаний пластины.

В случае вибрирующего стержня на свободной поверхности жидкости взаимодействие стержня с жидкостью ограничивается площадью медиального сечения стержня, что позволяет достигать больших амплитуд вибраций по сравнению с вибрациями пластины в жидкости.

С увеличением амплитуды вибраций пластины происходит параметрическое возбуждение капиллярных колебаний в виде поперечных капиллярных стоячих волн на поверхности смачивающего слоя жидкости вблизи края линии смачивания поверхности пластины жидкостью. Пучности в этих капиллярных волнах вытянуты в направлении, поперечном линии смачивания (рис. 7, $c$ и f). Так как в пучностях стоячей волны радиус кривизны в поперечном направлении меньше радиуса кривизны в продольном направлении, скорость волны должна быть больше в поперечном направлении. Стоячая волна представляет собой суперпозицию бегущих волн, движущихся в противоположных направлениях. В связи с этим в области формирования стоячей волны величина отрицательного давления в среднем в жидкости увеличивается и жидкость движется в эту область. Это приводит к формированию потока, направленного в сторону поверхности вибрирующей пластины. Этот поток, в свою очередь, разделяется и ускоряется бегущими в противоположных направлениях капиллярными волнами.

Рассмотренный механизм формирования течений вибрирующей пластиной возможен также в тонких слоях и каплях жидкости на вибрирующей подложке при возбуждении двумерных капиллярных волн на поверхности слоя или капли жидкости. Пучности в двумерных капиллярных колебаниях являются общими для волн, распространяющихся во взаимно поперечных направлениях с различными волновыми векторами.

\section{Выводы}

Проведенный анализ показывает, что среднее давление, создаваемое в жидкости вибрирующей пластиной вблизи ее поверхности, является отрицательным. Вследствие этого вдоль свободной поверхности жидкости между невозмущенной и возмущенной областями возникает градиент давления, приводящий жидкость в движение к краям пластины в поперечном направлении к плоскости колебаний. Таким образом, формируются два потока, направленных к краям пластины. Непосредственно вблизи пластины каждый из потоков разделяется капиллярными колебаниями в виде пучностей на поверхности, периодически создаваемых на противоположных сторонах пластины. Смещение потоков происходит за счет импульса капиллярных волн. В свою очередь, два потока с одной и той же стороны пластины объединяются в поток, направленный в плоскости колебаний, и ускоряются капиллярными волнами. В результате этого при вибрациях пластины в жидкости на ее поверхности формируются четыре вихревых плоских течения.

\section{Финансирование работы}

Работа выполнена при частичной поддержке гранта РФФИ № 16-41-180276_p_урал_а.

\section{Список литературы}

[1] Faraday M. // Philosophical Transactions of the Royal Society of London. 1831. Vol. 121. P. 299-340.

DOI: http://www.jstor.org/stable/107936

[2] Périnet N., Gutiérrez P., Urra H., Mujica N., Gordillo L. // J. Fluid Mech. 2017. Vol. 819. P. 285-310.

DOI: $10.1017 / \mathrm{jfm} .2017 .166$

[3] Филатов С.В., Бражнников М.Ю., Левченко А.А. // Письма в ЖЭТФ. 2015. Т. 102. Вып. 7. С. 486-490. [Filatov S.V., Brazhnikov M.Yu., Levchenko A.A. // JETP Lett. 2015. Vol. 102. N 7. P. 432-436.] DOI: https://doi.org/10.1134/S0021364015190054

[4] Afenchenko V.O., Ezersky A.B., Kiyashko S.V., Rabinovich M.I., Weidman P.D. // Phys. Fluid. 1998. Vol. 10. N 2. P. 390-399. DOI: $10.1063 / 1.869571$

[5] Зейтунян P.Х. // УФН. 1998. Т. 168. № 3. С. 259-386. DOI:10.3367/UFNr.0168.199803b.0259

[6] Лебедев-Степанов П.В., Руденко О.В. // Акустический журнал. 2016. Т. 62. № 4. С. 408-411. DOI:10.7868/S0320791915020094

[7] Aleksandrov V.A., Kopysov S.P., Tonkov L.E. // Microgravity Sci. Technol. 2018. Vol. 30. N 1-2. P. 85-93. DOI: https://doi.org/10.1007/s12217-017-9579-0 